\title{
Irrigation port hydration in phacoemulsification surgery
}

This article was published in the following Dove Press journal: Clinical Ophthalmology

\author{
Hisaharu Suzuki' \\ Yoichiro Masuda ${ }^{2}$ \\ Yuki Hamajima' \\ Hiroshi Takahashi ${ }^{3}$ \\ 'Department of Ophthalmology, \\ Nippon Medical School Musashikosugi \\ Hospital, Kawasaki City, Kanagawa, \\ ${ }^{2}$ Department of Ophthalmology, \\ The Jikei University, Katsushika \\ Medical Center, Tokyo, ${ }^{3}$ Department \\ of Ophthalmology, Nippon Medical \\ School, Tokyo, Japan
} using an irrigation port.
Background: In most cases, hydration is performed by water injection into the stromal tissue with a needle. The technique is simple, however it is sometimes troublesome.

Purpose: We describe a simple technique for hydrating the corneal stroma in cataract surgery

Patients and methods: The technique began by pushing the irrigation port against the corneal stroma for a few seconds during phacoemulsification, which generated edema in the corneal incision that subsequently prevented leakage. This procedure is called the hydration using irrigation port (HYUIP) technique. A total of 60 eyes were randomized and placed in two groups, 30 eyes underwent surgeries using the HYUIP technique (HYUIP group) and 30 eyes underwent surgeries without the HYUIP technique (control). The three points evaluated during each surgery included 1) the occurrence of anterior chamber collapse during the pulling out of the I/A tip after inserting the intraocular lens, 2) the need for conventional hydration, and 3) watertight completion at the end stage of surgery.

Results: The anterior chamber collapse and the need for conventional hydration were significantly smaller in the HYUIP group compared to the control group. Regarding the self-sealing completion, no significant difference was observed between the two groups.

Conclusion: The HYUIP technique is an effective method for creating self-sealing wound. In addition, this technique helps to prevent anterior chamber collapse.

Keywords: cataract surgery, hydration, irrigation and aspiration, phacoemulsification, wound, self-sealing

\section{Introduction}

Today, most phacoemulsifications are sutureless surgeries in which the wound is self-sealing. The most common technique utilized for creating this wound is through the use of stromal hydration. Compromised wound integrity in the form of fluid leakage, which can induce bacterial invasion from the ocular surface, is believed to be a risk factor for endophthalmitis. ${ }^{1}$ Therefore, creating a watertight wound is an important and necessary step for the safety of the procedure. When extracting the irrigation/aspiration (I/A) tip after the insertion of the intraocular lens, opening of the wound often occurs, thereby leading to collapse of the anterior chamber. Consequently, surgeons need to hydrate the wound site stroma to make the wound watertight and reconstruct the anterior chamber integrity. In most cases, hydration is performed by water injection into the stromal tissue with a needle. The technique is simple, but is sometimes troublesome. Here, we report a very simple technique that can be used to carry out hydration prior to pulling out the tip from the anterior chamber. 


\section{Patients and methods}

This study was a randomized controlled trial. Approval was obtained from the Institutional Review Board/Ethics Committee of Nippon Medical School Musashikosugi Hospital prior to the start of the study. Before enrollment to the study, written informed consent was obtained from all patients. From December 2016 through February 2017, one surgeon (HS) performed all cataract surgeries at Nippon Medical School Hospital Musashikosugi Hospital. All incisions were created just anterior to the transconjunctival single-plane sclerocorneal incision $^{2}$ using a $2.4 \mathrm{~mm}$ wide steel keratome. The planned length of all incisions was approximately $2.0 \mathrm{~mm}$ with no enlargement. We used a Stellaris phaco machine (Bausch + Lomb, Aliso Viejo, CA, USA) in all cases. Phacoemulsification was performed through a superior transconjunctival single-plane sclerocorneal incision using an ophthalmic viscosurgical device of sodium hyaluronate $2.3 \%$ (Healon5; Allergan Medical Optics, Santa Ana, CA, USA), an ultrasound power output of $20 \%$, and a vacuum pressure of $150 \mathrm{mmHg}$. The bottle height setting was $70 \mathrm{~cm}$. Following phacoemulsification and cortical cleanup, a 1-piece foldable acrylic intraocular lens was inserted via an injector through the wound. After removing the ophthalmic viscosurgical device from the anterior chamber, the irrigation port of the I/A tip was used to press the upper, lower, and lateral sides of the wound in order to create hydration for the corneal stroma. The pushing time for each side was 5-10 seconds (Figure 1). Then, once the chip was returned to the anterior chamber, it was quickly pulled out. A Medical Quick Absorber was subsequently used to confirm whether there was leakage from the wound (Figure 2). This procedure is referred to as the hydration using irrigation port (HYUIP) technique.

A total of 60 eyes were randomized and placed in two groups, with 30 eyes undergoing surgeries using the HYUIP technique (HYUIP group) and 30 undergoing surgeries without the HYUIP technique (control). Randomization was done
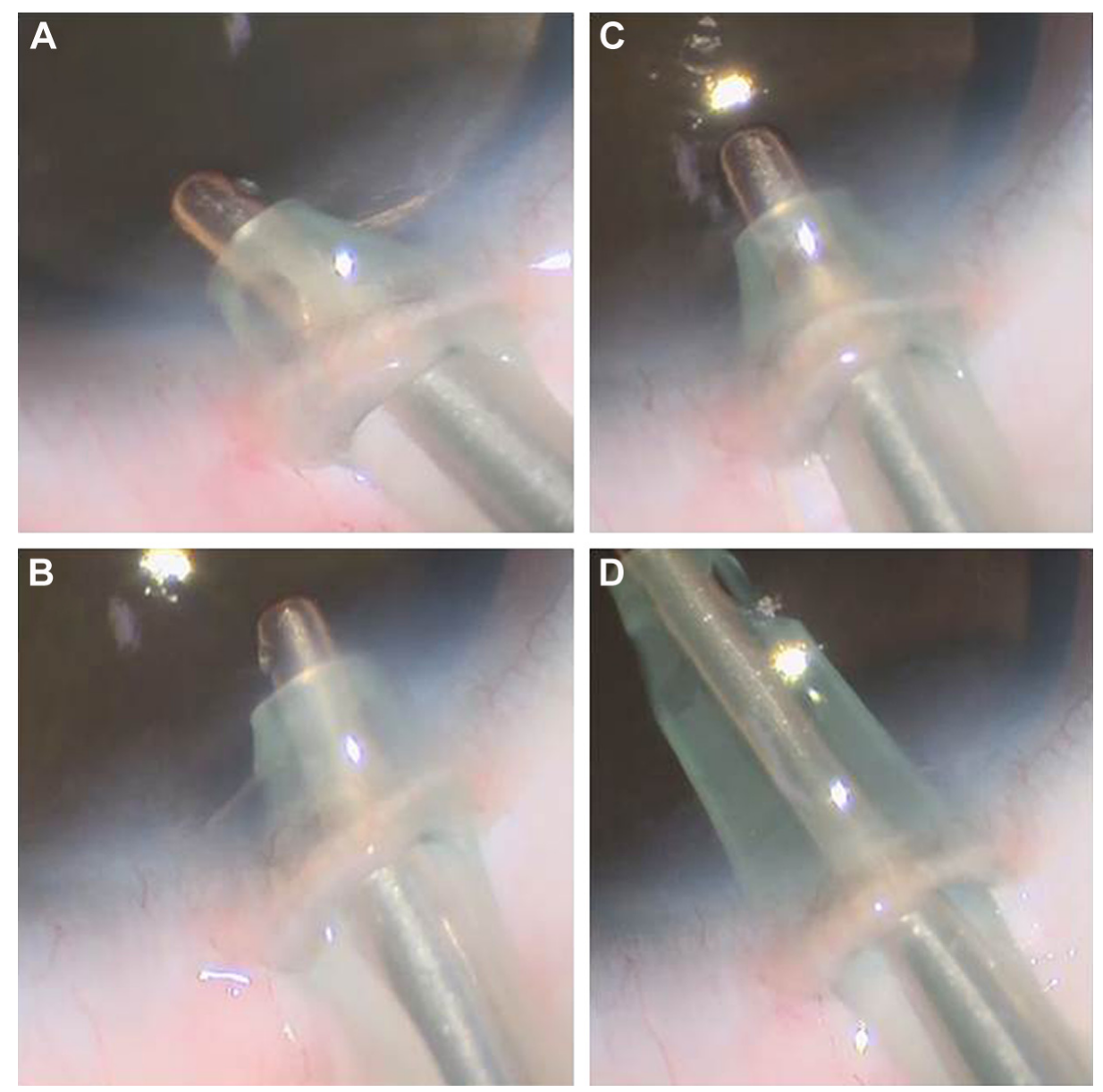

Figure I HYUIP technique I.

Notes: (A) The irrigation port of the tip was used to push the left side of the corneal incision for 5-10 seconds. (B) The procedure listed in part A was repeated for the right side. (C) The procedure listed in part A was repeated in the up and down directions. (D) After pushing the three sides, the tip was immediately drawn back inside the anterior chamber.

Abbreviation: HYUIP, hydration using irrigation port. 

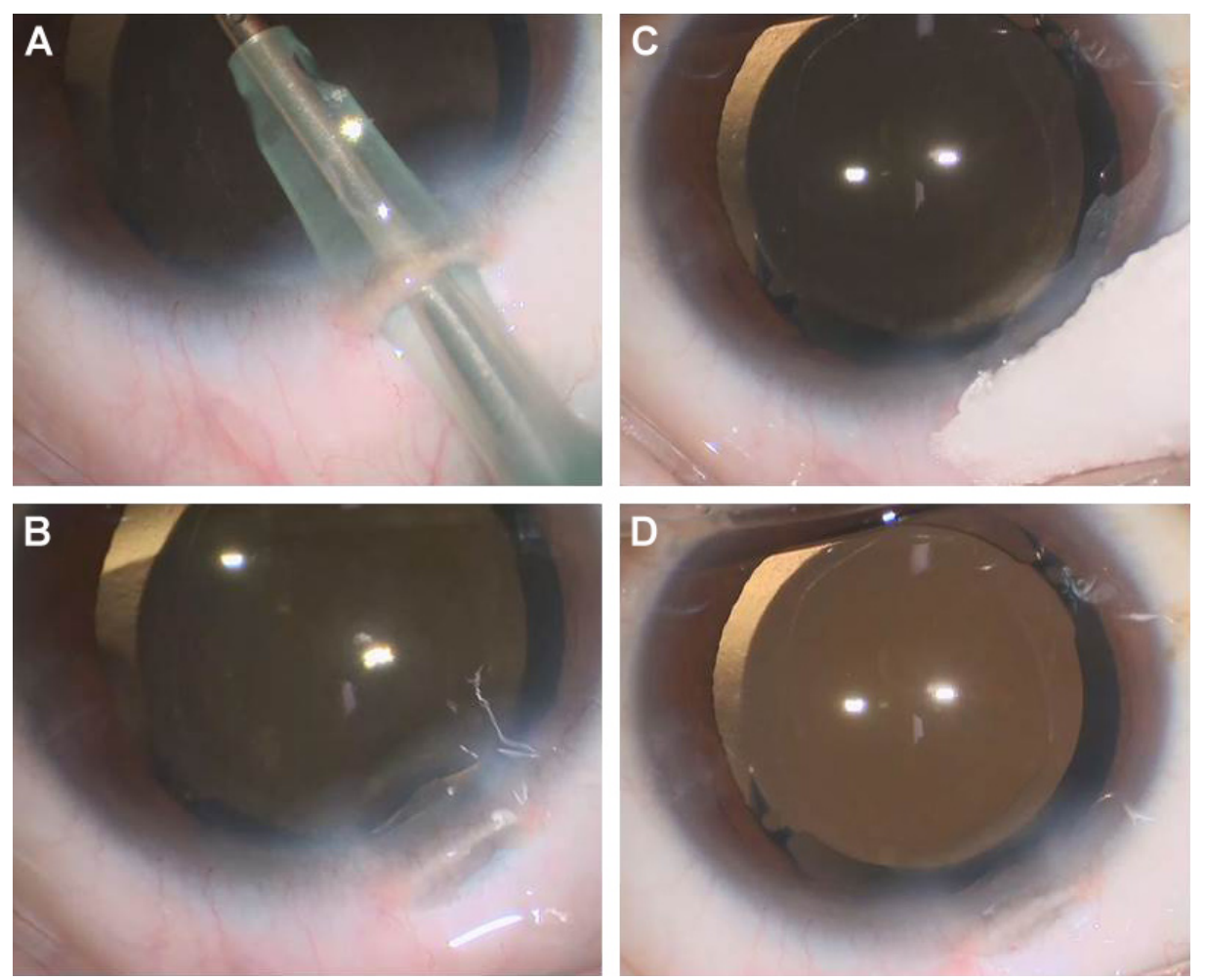

Figure 2 HYUIP technique 2.

Notes: (A) The tip was maintained in place until the anterior chamber depth stabilized. (B) When the tip was removed, it was pulled from the anterior chamber using a single stroke. (C) MQA was used to confirm the presence of any leakage from the incisional wound. (D) After extracting the tip, the anterior chamber depth was maintained without any further changes.

Abbreviations: HYUIP, hydration using irrigation port; MQA, Medical Quick Absorber.

by the envelope method. The three points evaluated during each surgery included 1) the occurrence of anterior chamber collapse during the pulling out of the I/A tip after inserting the intraocular lens, 2) the need for conventional hydration, and 3) watertight completion at the end stage of surgery. The data in the two groups were compared using Fisher's test. Any differences with a $P$-value of $<0.01$ were considered statistically significant. In addition, the wound integrity was observed in eyes in both groups using the Rescan 700 OCT device (Zeiss, Jena, Germany).

Morphology of the central corneal endothelium was analyzed using a specular microscope, and cell density, coefficient of variation, and percentage of the hexagonal cell were determined. Statistical analysis was performed using the unpaired Student's $t$-test. All $P$-value $<0.01$ were considered significant. Excel (Microsoft, Redmond, WA, USA) was used for statistical analysis.

\section{Results}

The clinical study comprised 60 patients (60 eyes); there were 30 patients in the HYUIP group and 30 patients in the control group. In all cases, the surgery lasted fewer than
10 minutes and the effective phaco time was generally fewer than 10 seconds.

Table 1 summarizes the findings for the occurrence of anterior chamber collapse, the need for conventional hydration, and the watertight completion at the end stage of surgery. The anterior chamber collapse and the need for conventional hydration were significantly smaller in the HYUIP group compared to the control group. Regarding the self-sealing completion, no significant difference was observed between the two groups. Optical coherence tomogrpahy (OCT) images were obtained during the phacoemulsification using the Rescan 700 OCT device (Zeiss) in both groups. In the HYUIP group, images included the area around the self-sealing wound that was created using an

Table I Comparison between the two groups

\begin{tabular}{llll}
\hline Evaluation items & $\begin{array}{l}\text { HYUIP } \\
\text { (30 eyes) }\end{array}$ & $\begin{array}{l}\text { Control } \\
\text { (30 eyes) }\end{array}$ & P-value \\
\hline Anterior chamber collapse & 4 & 24 & $<0.0 I^{*}$ \\
Conventional hydration & 0 & 24 & $<0.0 I^{*}$ \\
Posthydration suture & 0 & 0 & 1.0 \\
\hline
\end{tabular}

Note: *Statistically significant difference (Fisher's test).

Abbreviation: HYUIP, hydration using irrigation port. 

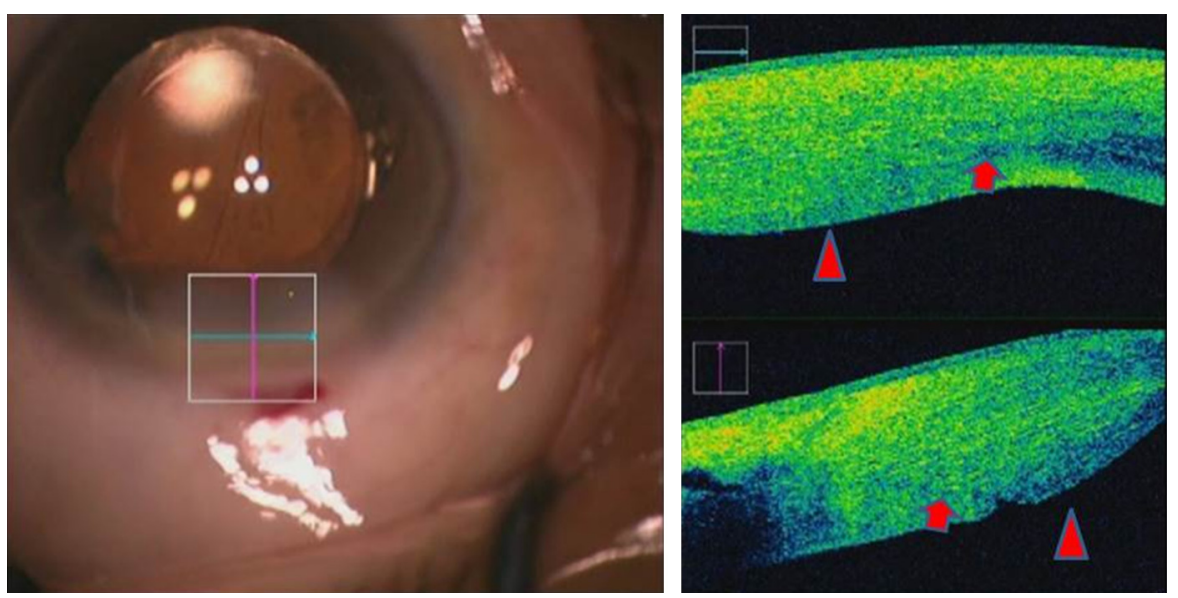

Figure 3 OCT images of the HYUIP technique group.

Notes: Red arrows indicate the corneal incisional wound. Red triangles indicate corneal stromal edema. Images show that the incision layer was self-sealing due to the presence of edema at both the left and right edges.

Abbreviations: HYUIP, hydration using irrigation port; OCT, optical coherence tomography.

irrigation port (Figure 3). In control, the degree of corneal edema was greater than HYUIP. However, there were some cases in which slight detachment of Descemet's membrane was observed around the incision (Figure 4). All patients in both groups exhibited excellent vision, with corrected visual acuity being $0.1 \log$ MAR or better, and experienced no complications after the surgery. There was no significant difference in degree of decrease (\%) in cell density at 1-month postop between 2 groups (Figure 5); also, no significant difference was observed in the coefficient of variation and the hexagonal cell ratio between 2 groups.

\section{Discussion}

Sutureless cataract surgery has now become a standard technique. However, there is concern that endophthalmitis could occur after these surgeries. During cataract surgery, it is well known that there are essentially two opportunities for bacteria to enter the eye. The first possibility of entry occurs at the time of surgery, while the second opportunity is during the early postoperative period prior to the epithelialization of an unsutured wound. To prevent invasion of bacteria, stromal hydration has been the most common technique used to augment the self-sealing wound dynamics of corneal incisions. To hydrate a wound, water injection using a needle is the most popular method. However, when using a needle, there are potential complications that need to be taken into consideration. Bradshaw et $\mathrm{al}^{3}$ reported that use of a slip-lock cannula during the stromal hydration step of what was otherwise a routine cataract procedure resulted in a case of iris perforation, zonule rupture, hyphema, and vitreous
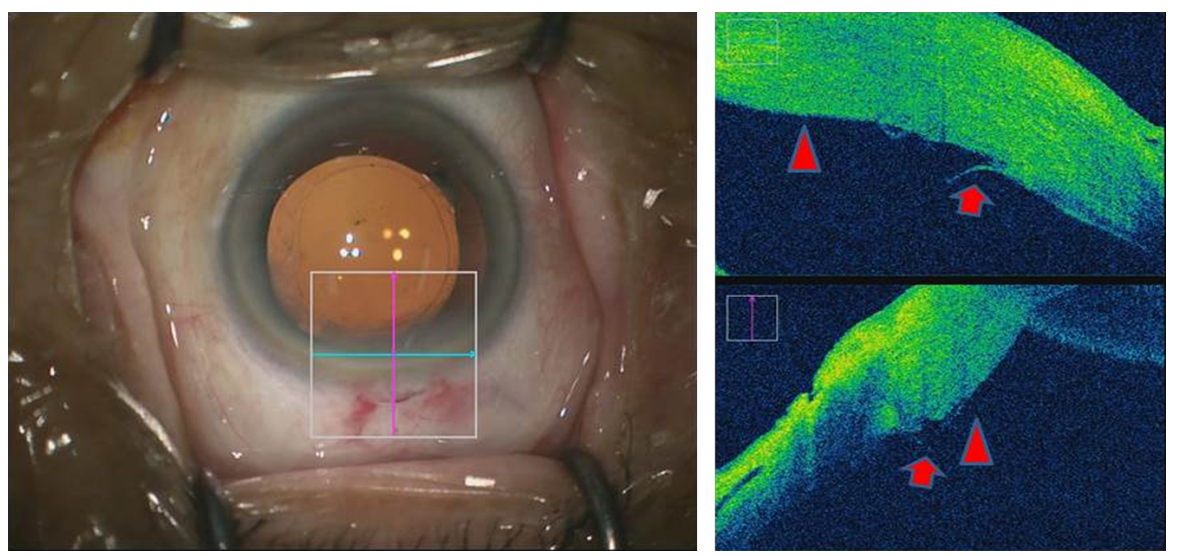

Figure 4 OCT images of normal hydration.

Notes: Red arrows indicate slight detachment of Descemet's membrane. Red triangles indicate corneal stromal edema. Images show that the incision layer was self-sealed due to the presence of edema around all the incision.

Abbreviation: OCT, optical coherence tomography. 


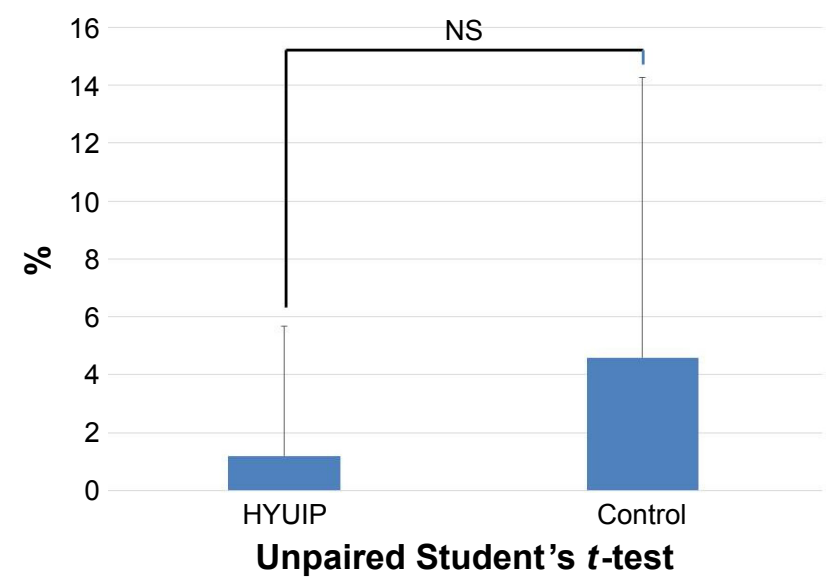

Figure 5 Degree of decrease (\%) in cell density (mean \pm SD). Abbreviations: HYUIP, hydration using irrigation port; NS, not significant; SD, standard deviation.

hemorrhage. Further studies using OCT analysis revealed that the clear corneal incision with stromal hydration showed local detachment of Descemet's membrane. ${ }^{4,5}$ Calladine et $\mathrm{al}^{4}$ reported that wound hydration was more likely to cause a localized Descemet's membrane detachment compared to wounds without hydration ( $65 \%$ vs $25 \%$ ). Thus, it is possible that these complications could be associated with the use of a needle during the procedure. The cause of these complications could be related to the possibility that the use of a needle results in a concentration of pressure in one position between the stroma and Descemet's membrane. Sun et al ${ }^{6}$ examined porcine eyes and reported finding a pressure that was beyond $140 \mathrm{psi}$ (maximum range), which far exceeded the pressure required to hydrate the stroma.

In order to avoid these complications, several methods have been suggested to ensure there is proper sealing of the incision at the end of surgery. Nithyanandarajah et $\mathrm{al}^{7}$ reported a technique for sealing clear corneal incisions using a 30-gauge needle and injection of a balanced salt solution into the anterior stroma above the incision. Mifflin ${ }^{8}$ described finding that the anterior stromal pocket hydration technique was significantly better than conventional hydration in preventing a wound leak due to the direct pressure on the posterior lip of the incision. Thus, it appears that these procedures are effective in terms of strengthening the construction of the incision wounds. However, since these procedures are all carried out at the end of the surgeries, they do not offer any great effect in helping to prevent anterior chamber collapse. In contrast, the HYUIP technique is carried out prior to pulling the tip from the anterior chamber. Thus, this technique not only constructs a self-sealing wound but also prevents the collapse of the anterior chamber. It may be useful to prevent rotation of the toric intraocular lens because the rotation has been reported to be associated with the collapse of the anterior chamber immediately after the surgery. ${ }^{9}$ Furthermore, since the pressure is not concentrated in one part when using this technique, it is possible that this procedure will also prevent excessive invasion of the corneal stroma and other complications such as local detachment of Descemet's membrane.

It should be noted, however, that it is possible that the hydration pressure when using this technique will be weaker than that found for the conventional method. Therefore, when the length of the wound is short and there is only a slight thermal burn injury, it is possible that these incisions could be difficult to close. However, in our current study, we found that this technique was very effective in all patients who underwent the procedure. Use of OCT imaging during the surgery made it possible to delineate the edema around the incisional wound and confirm that this effect contributed to the creation of a self-sealing wound. These observations strengthen our assertion that the HYUIP technique is effective in normal cases undergoing this type of procedure.

\section{Conclusion}

In conclusion, the HYUIP technique is an effective method for creating a self-sealing wound. In addition, this technique helps to prevent anterior chamber collapse.

\section{Acknowledgment}

We thank Kotaro Oki and Hisaharu Iwaki for their helpful suggestions regarding this technique.

\section{Disclosure}

The authors report no conflicts of interest in this work.

\section{References}

1. Vasavada AR, Praveen MR, Pandita D, et al. Effect of stromal hydration of clear corneal incisions: quantifying ingress of trypan blue into the anterior chamber after phacoemulsification. J Cataract Refract Surg. 2007;33:623-627.

2. Sugai S, Yoshitomi F, Oshika T. Transconjunctival single-plane sclerocorneal incisions versus clear corneal incisions in cataract surgery. J Cataract Refract Surg. 2010;36:1503-1507.

3. Bradshaw SE, Shankar P, Maini R, Ragheb S. Ocular trauma caused by a loose slip-lock cannula during corneal hydration. Eye. 2006;20(12): 1432-1434.

4. Calladine D, Tanner V. Optical coherence tomography of the effects of stromal hydration on clear corneal incision architecture. J Cataract Refract Surg. 2009;35(8):1367-1371

5. Marcon AS, Rapuano CJ, Jones MR, Laibson PR, Cohen EJ. Descemet's membrane detachment after cataract surgery: management and outcome. Ophthalmology. 2002;109(12):2325-2330.

6. Sun MT, Wood M, Chan W, Casson R. Pressures generated during corneal wound hydration. J Cataract Refract Surg. 2016;42(9):1383-1384. 
7. Nithyanandarajah G, Athanasiadis Y, Scollo P, Sharma B, Dorgham A, Sharma A. Hydration of the anterior stroma in phacoemulsification cataract surgery. J Cataract Refract Surg. 2014;40:702-704.

8. Mifflin MD, Kinard K, Neuffer MC. Comparison of stromal hydration techniques for clear corneal cataract incisions: conventional hydration versus anterior stromal pocket hydration. J Cataract Refract Surg. 2012; 38(6):933-937.
9. Inoue Y, Takehara H, Oshika T. Axis misalignment of toric intraocular lens: placement error and postoperative rotation. Ophthalmology. 2017; 124(9):1424-1425.

\section{Publish your work in this journal}

Clinical Ophthalmology is an international, peer-reviewed journal covering all subspecialties within ophthalmology. Key topics include: Optometry; Visual science; Pharmacology and drug therapy in eye diseases; Basic Sciences; Primary and Secondary eye care; Patient Safety and Quality of Care Improvements. This journal is indexed on

\section{Dovepress}

PubMed Central and CAS, and is the official journal of The Society of Clinical Ophthalmology (SCO). The manuscript management system is completely online and includes a very quick and fair peer-review system, which is all easy to use. Visit http://www.dovepress.com/ testimonials.php to read real quotes from published authors. 